\title{
Lesson of the month: Recurrent falls and hypoglycaemia in an older woman
}

\author{
Authors: Alvin SQ Ong, ${ }^{A}$ Lin Wang ${ }^{\mathrm{B}}$ and Junjie $\mathrm{Aw}^{\mathrm{C}}$
}

\begin{abstract}
We present a case of a non-diabetic 84-year-old woman who presented with recurrent falls and hypoglycaemic episodes and was subsequently diagnosed to have a solitary insulinoma. Her multiple fall episodes may have been precipitated by her low blood glucose levels. We discuss the clinical and radiological findings of insulinoma.
\end{abstract}

KEYWORDS: falls, hypoglycaemia, insulinoma

DOI: $10.7861 /$ clinmed.2021-0671

\section{Case presentation}

An 84-year-old woman was admitted to hospital in view of a near fall after a recent admission for small bowel obstruction secondary to right hernia, for which a diagnostic laparoscopy, mini laparotomy, adhesiolysis and an open right inguinal hernia mesh repair was done. Prior to the fall, patient did not report any symptoms of giddiness, headache, blurring of vision, focal numbness or weakness, chest pain or shortness of breath. During the fall, there was no loss of consciousness or abnormal jerking movement of limbs. The near fall episode was attributed to various degenerative bone and joint diseases.

She was subsequently transferred to a community hospital for further rehabilitation. During her stay, she was found to have recurrent hypoglycaemia which typically occurred in the morning. During her episodes of hypoglycaemia, she remained asymptomatic. Her renal function, liver panel, infection markers, thyroid function and cortisol level were normal, as was a drug screening test. Her serum C-peptide was 1.20 (reference range $0.78-5.19 \mathrm{ug} / \mathrm{L}$ ), serum insulin was 0.26 (reference range $1.0-30.0$ $\mathrm{mU} / \mathrm{L}$ ) and fasting plasma glucose was 2.3 (reference range 3.9$6.0 \mathrm{mmol} / \mathrm{L}$ ). Previous CT abdomen pelvis (Fig 1) done for intestinal obstruction was found to have a dense focus in the periampullary area which was either duodenal or pancreatic in origin. The lesion was further characterised by an MRI pancreas (Fig 2), which showed the presence of a pancreatic head lesion likely due to a

Authors: ${ }^{\text {A }}$ medical officer, Outram Community Hospital, Singapore; ${ }^{B}$ staff registrar, Outram Community Hospital, Singapore; ${ }^{C}$ associate consultant, Outram Community Hospital, Singapore; clinical instructor, DUKE-NUS Medical School, Singapore and clinical physician faculty member, SingHealth, Singapore

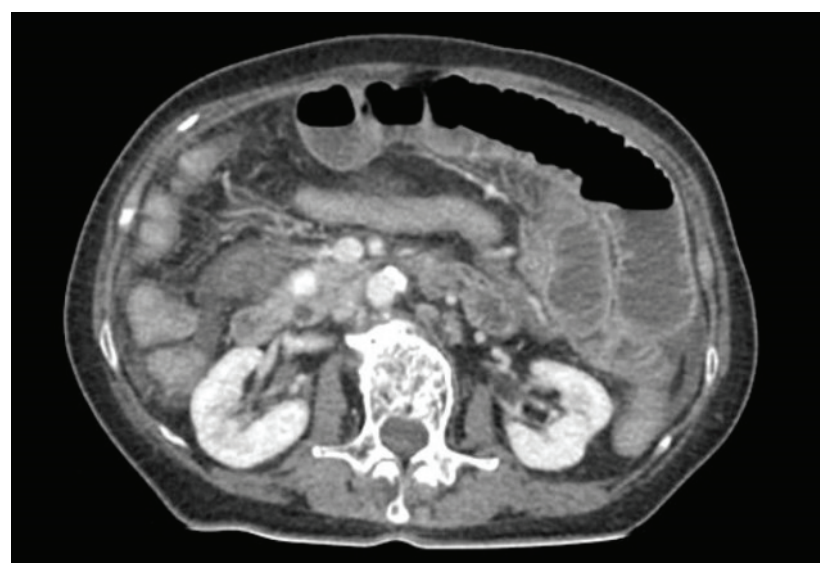

Fig 1. CT abdomen pelvis. Dense focus in periampullary region which was either duodenal or pancreatic in origin.

calcified insulinoma. Subsequently, a PET DOTATE scan (Fig 3) was performed with findings in keeping with a tracer-avid lesion in the pancreatic head, which may represent a neuroendocrine tumour. Endoscopic ultrasound-guided fine needle aspiration cytology was done and confirmed the diagnosis of neuroendocrine tumour. The patient was treated with diazoxide $100 \mathrm{mg} \mathrm{BD}$.

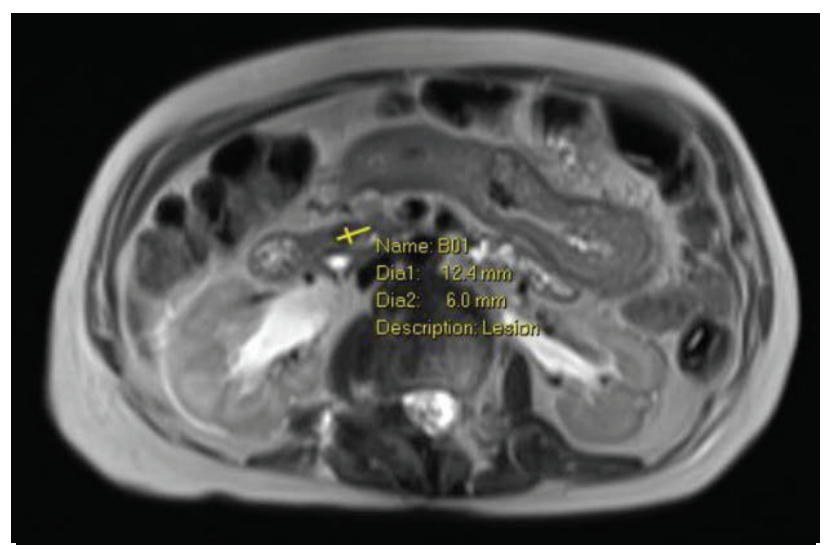

Fig 2. MRI pancreas. This shows a pancreatic head lesion which may represent a calcified insulinoma. 


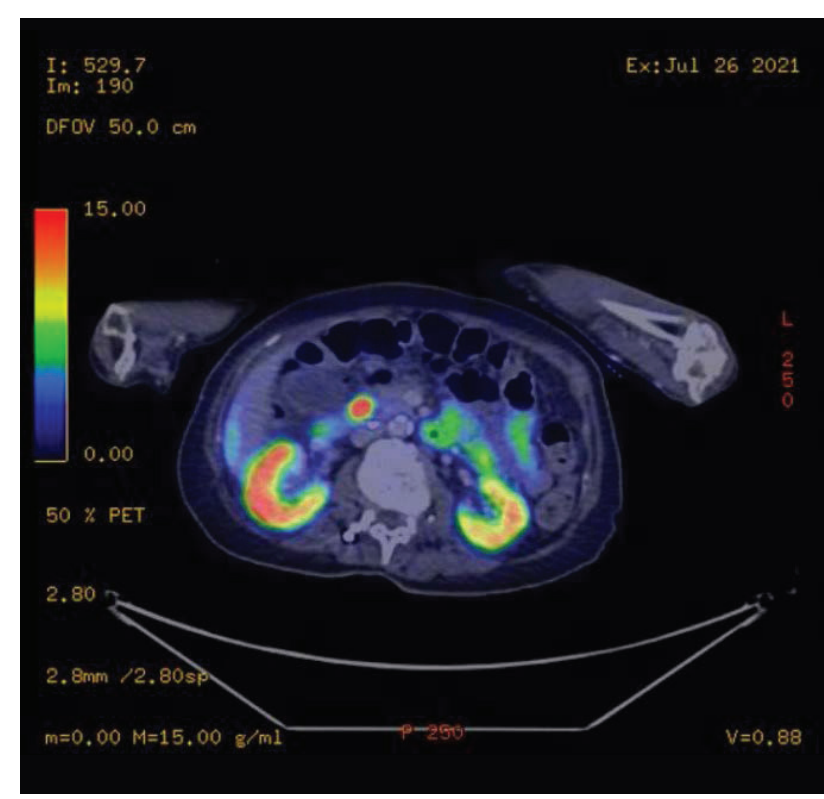

Fig 3. PET DOTATE scan. In the pancreatic head, adjacent to the distal common bile duct, there is a focal increase in tracer uptake, likely corresponding to known calcification demonstrated in prior MRI/CT study, SUVmax 23.3. The findings are suggestive of an insulinoma.

\section{Discussion}

Insulinomas may have varied presentations in the elderly population. In the above case example, one of the possible causes of her near fall event could be attributed to her hypoglycaemic episodes. Insulinoma has an annual incidence of four in 1 million persons and is considered the most common neuroendocrine tumour of the pancreas. ${ }^{1}$ In terms of the pathogenesis of insulinomas, they can occur sporadically or in conjunction with MEN-1 syndrome.

Non-diabetic patients with documented Whipple's triad or severely depressed serum glucose level $(<2.2 \mathrm{mmol} / \mathrm{L})$ require further evaluation, with differential diagnosis including insulinoma, drugs, critical illnesses, hormonal deficiency or paraneoplastic syndrome. The gold standard of biochemical diagnosis of insulinoma is to measure insulin, C-peptide, plasma glucose and proinsulin during a 72 -hour fast. ${ }^{2-4}$ Studies have reported that helical computerised tomography and endoscopic ultrasound can provide higher sensitivity ( $94 \%$ sensitivity in both). ${ }^{5}$ A DOTATATE PET/CT can also identify most insulinomas and can be used as an adjunct imaging study when other imaging studies are non-conclusive. ${ }^{6}$

Hypoglycaemia unawareness happens when recurrent hypoglycaemia shifts the glycaemic threshold for counterregulation, as in this case. Florid neurogenic symptoms are therefore blunted, while non-specific neuroglycopaenia signs and symptoms may predominate. ${ }^{7}$ Despite being reviewed by a dietician as an inpatient, the patient continued having hypoglycaemic events. A diagnosis of insulinoma was considered after thorough work up for hypoglycaemia. Localisation via radiological studies and tissue diagnosis was pursued. The median duration of symptoms before diagnosis of insulinoma has been shown in one study to be 18 months. ${ }^{8}$ A delayed diagnosis and treatment may be potentially dangerous for patients, whereas early diagnosis can prevent falls, syncope, cognitive impairment and improve quality of life for geriatric patients.

Insulinoma can be treated medically or surgically. The definitive treatment for benign insulinoma is surgery, although other techniques, such as injection of octreotide, endoscopicultrasound-guided alcohol ablation, radiofrequency ablation (RFA) and embolisation of an insulinoma of the pancreas, have been described. ${ }^{9,10}$ Our patient opted for medical treatment after discussing the pros and cons of the treatment options. No further hypoglycaemic episodes occurred afterwards.

\section{Conclusion}

We present a case of a non-diabetic elderly woman who presented with recurrent falls and hypoglycaemic episodes who was subsequently diagnosed to have a solitary insulinoma. Her multiple fall episodes may have been precipitated by her low blood glucose levels.

Patients and clinicians should engage in shared decision making on whether to pursue surgical or medical management.

\section{References}

1 Service FJ, McMahon MM, O'Brien PC, Ballard DJ. Functioning insulinoma - incidence, recurrence, and long-term survival of patients: a 60-year study. Mayo Clin Proc 1991;66:711-9.

2 Service FJ, Natt N. The prolonged fast. J Clin Endocrinol Metab 2000;85:3973-4.

3 Eichelberger GS, Carbono J, Field Z, Kainaur K, Montalvo F. Case report and literature review of insulinoma in the geriatric population: An 86-year-old female with syncope of unknown origin. Case Rep Endocrinol 2020;2020:8870776.

4 Vella A. Hypoglycemia in adults without diabetes mellitus: Diagnostic approach. www.uptodate.com/contents/hypoglycemiain-adults-without-diabetes-mellitus-diagnostic-approach [Accessed 26 September 2021].

5 McAuley G, Delaney H, Colville ] et al. Multiomdality preoperative imaging of pancreatic insulinomas. Clin Radiol 2005;60:1039-50.

6 Nockel P, Babic B, Millo C et al. Localization of insulinoma using 68Ga-DOTATE PET/CT Scan. J Clin Endocrinol Metob 2017;102: 195-9.

7 Tesfaye N, Seaquist ER. Neuroendocrine responses to hypoglycemia. Ann N Y Acad Sci 2010;1212:12-28.

8 Bouslama K, Maghrebi H, Bedioui H, Bouslama K. [Pancreatic insulinoma: diagnostic approach and therapeutic modalities.] ] Afr Hepato-Gastroenterol 2014;8:11-5.

9 Rott G, Biggemann M, Pfohl M. Embolization of an insulinoma of the pancreas with trisacyl gelatin microspheres as definitive treatment. Cardiovasc Intervent Radiol 2008;31:659-62.

10 Jürgensen C, Schuppan D, Neser F, Ernstberger J, Junghans U, Stölzel U. EUS-guided alcohol ablation of an insulinoma. Gastrointest Endosc 2006;63:1059-62.

Address for correspondence: Dr Alvin SQ Ong, Outram Community Hospital, Post-Acute and Continuing Care Department, 10 Hospital Boulevard, Singapore 168582, Singapore.

Email: alvinong@gmail.com 